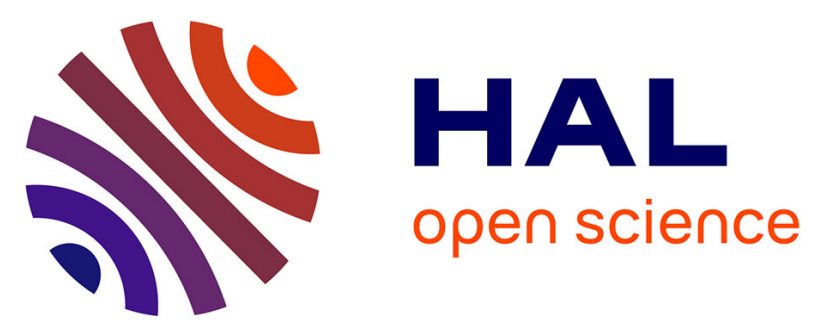

\title{
Combination treatment of resveratrol and capsaicin radiosensitizes pancreatic tumor cells by unbalancing DNA repair response to radiotherapy towards cell death
}

Véronique Vendrely, Samuel Amintas, Cécile Noel, Isabelle Moranvillier, Isabelle Lamrissi, Benoit Rousseau, Soulé Coulibaly, Aurélie Bedel, François Moreau-Gaudry, Etienne Buscail, et al.

\section{To cite this version:}

Véronique Vendrely, Samuel Amintas, Cécile Noel, Isabelle Moranvillier, Isabelle Lamrissi, et al.. Combination treatment of resveratrol and capsaicin radiosensitizes pancreatic tumor cells by unbalancing DNA repair response to radiotherapy towards cell death. Cancer Letters, 2019, 451, pp.1 - 10. 10.1016/j.canlet.2019.02.038 . hal-03486867

\section{HAL Id: hal-03486867 https://hal.science/hal-03486867}

Submitted on 20 Dec 2021

HAL is a multi-disciplinary open access archive for the deposit and dissemination of scientific research documents, whether they are published or not. The documents may come from teaching and research institutions in France or abroad, or from public or private research centers.
L'archive ouverte pluridisciplinaire HAL, est destinée au dépôt et à la diffusion de documents scientifiques de niveau recherche, publiés ou non, émanant des établissements d'enseignement et de recherche français ou étrangers, des laboratoires publics ou privés.

\section{(ㄷ)(1) $\$$}

Distributed under a Creative Commons Attribution - NonCommerciall 4.0 International 
Version of Record: https://www.sciencedirect.com/science/article/pii/S0304383519301247

Manuscript_6d3500c4b268880898caac0b9b8f1f2d

Combination treatment of resveratrol and capsaicin radiosensitizes pancreatic tumor cells by unbalancing DNA repair response to radiotherapy towards cell death

Véronique Vendrely ${ }^{1,2}$, Samuel Amintas ${ }^{2,3}$, Cécile Noel $^{3}$, Isabelle Moranvillier ${ }^{1,3}$, Isabelle Lamrissi ${ }^{1,3}$, Benoit Rousseau ${ }^{3}$, Soulé Coulibaly ${ }^{2}$, Aurélie Bedel ${ }^{1,2,3}$, François Moreau-Gaudry ${ }^{1,2,3}$, Etienne Buscail ${ }^{1,2,3}$, Laurence

Chiche $^{1,2,3}$, Geneviève Belleannée ${ }^{2}$, Charles Dupin ${ }^{2}$ and Sandrine Dabernat ${ }^{1,2,3, \&}$

${ }^{1}$ INSERM U1035, Bordeaux, France

${ }^{2} \mathrm{CHU}$ de Bordeaux, Bordeaux, France

${ }^{3}$ Université de Bordeaux, Bordeaux, France

Running title: BFC combination with radiotherapy in pancreatic cancer

\&Corresponding author:

Université de Bordeaux

INSERM U1035

146, rue Léo Saignat

33076 Bordeaux Cedex

Email: sandrine.dabernat@u-bordeaux.fr

Phone: +33(0) 557571374

Fax: +33(0) 557571374 


\section{ABSTRACT}

Pancreatic ductal adenocarcinoma (PDAC) remains one of the deadliest cancers because it is highly resistant to every available therapeutic strategy, in particular conventional chemotherapy or radiotherapy (RT). Sensitizing tumor cells to existing treatments remains a good option to obtain fast and applicable results. Considering that ionizing radiations induce radiolysis-derived reactive oxygen species (ROS), we hypothesized that ROS-inducing bioactive food components (BFCS) could exacerbate ROSrelated cell damages, including DNA double stranded breaks (DSBs), leaving the cellular ROS scavenging systems overwhelmed, and precipitating tumor cell death. Combination of resveratrol and capsaicin radiosensitized radiosensitive tumor cells, but RT did not increase BFC combination toxicity in radioresistant tumor cells. BFC addition to RT increased ROS production and led to significant tumor volume reduction in xenografted mouse preclinical model. Strikingly, BFCs inhibited RT-induced DNA damage molecular response by strongly limiting the first steps of DSB repair, and by keeping cells in cell cycle, provoking exacerbated intrinsic apoptosis. This study positions BFCs as potent radiosensitizers when combined, and identifies an actionable molecular pathway by resveratrol and capsaicin combination.

\section{HIGHLIGHTS}

Resveratrol and capsaicin used in combination increase radiosensitivity of pancreatic tumor cells.

Combined Bioactive Food Components (BFCs) addition to radiotherapy increases radio-induced reactive oxygen species.

BFCs combination with radiotherapy was more potent to limit tumor progression than radiotherapy alone.

Resveratrol and capsaicin strongly inhibit DNA double stranded break repair induced by radiotherapy.

BFCs might be an option as nutrition complements to radiosensitize pancreatic adenocarcinoma. 
Keywords : Resveratrol, Capsaicin, Radiotherapy, Pancreatic Ductal Adenocarcinoma, DNA repair

\section{Abbreviations}

PDAC: Pancreatic ductal adenocarcinoma

BFCs: Bioactive Food Components or Compounds

ROS: Reactive Oxygen Species

CSC: Cancer Stem Cell

FOLFIRINOX: Association of Oxaliplatin, Irinotecan and 5 Fluorouracile

IC50: Half maximal inhibitory concentration

C: Capsaicin

R: Resveratrol

RT: Radiotherapy

DSBs: Double Stranded Breaks

5FU: 5 Fluoro-Uracile

ATM: Ataxia Telangiectasia Mutated

Chk: checkpoint kinase

EMT: Epithelial to Mesenchymal Transition

ATR: ATM and Rad3-related

Con: control treated with vehicle

\section{ACKNOWLEDGEMENTS}


The authors would like to thank Katia Obama and Isabelle Lamy for administrative assistance and fund management, and the staff of University of Bordeaux animal facility, in particular Julien Izotte for technical assistance.

\section{FUNDING SOURCES}

This work was supported by grants from the Ligue régionale contre le cancer (Comité Dordogne), France.

\section{INTRODUCTION:}

Pancreatic ductal adenocarcinoma (PDAC) is associated with a poor prognosis, since the 5-year global survival rate remains under $10 \%$ (1). Given an increased incidence over the last decades, PDAC is becoming a major public health issue and is projected to become the second cancer killer in 2030 (2). This poor prognosis can be explained by the late diagnosis due to aspecific symptomatology but also by PDAC resistance towards conventional treatments as chemotherapy and radiotherapy (3). Such innate and acquired resistances most probably come from the multiple genomic aberrations found in PDAC. Indeed, more than 60 different mutations and 10 dysfunctional pathways have been identified by large scale high-throughput analyses(4), conferring multiple survival options to the tumor cells. Initially based on knowledge of PDAC, studies have tested clinically or are still evaluating radiosensitizing compounds inhibiting key oncogenic cell pathways such as ras, raf, EGFR and IGFR1 or modulating immune environment, with conflicting results or toxic issues(5). Thus, no specific radiosensitizers is currently admitted for PDAC, apart from 5-fluorouracil (5-FU) or gemcitabine, albeit with very limited success(6). Exposure to ionizing radiations induces a variety of DNA lesions, of which DNA double-stranded breaks (DSB) are the most lethal, and, if left unrepaired, lead to genomic instability or cell death. This is why several pathways have been proposed to contribute to radioresistance, mostly involved in DNA repair (7). Thus, it is very worthy to target DNA damage/repair pathways to increase RT-induced cell death. As 
conventional therapeutic approaches have been disappointing in PDAC, we looked for alternatives in the emerging field of BFCs. BFCs are physiologically active constituents in food or dietary supplements, including those that meet basic human nutrition needs, that have been demonstrated to have a role in health and to be safe for human consumption for intended food and dietary supplement uses (the American Dietetic Association). Among BFCs, resveratrol (a member of the stilbene family) and capsaicin (a vanilloid compound) had shown promising results on PDAC cell lines, by increasing Reactive Oxygen Species (ROS) production, and by potentiating chemo and/or radiotherapy (8). We found that combination of resveratrol and capsaicin potentiated a reduced dose of gemcitabine, resulting in full effect recovery of high dose of gemcitabine, in a preclinical mouse model (9). As BFCs, radiotherapy induces ROS production. Therefore, using several BFCs as an induction or a combination treatment with RT might overwhelm tumor cells in scavenging ROS, resulting in increased cell death. As we already showed that resveratrol and its analogues in combination with capsaicin increased chemotherapy toxicity, we ought to test whether this approach was valid for radiosensitization.

\section{MATERIALS AND METHODS}

\subsection{Animals and cell lines}

The 8 to 12 week-old Swiss Nude male mice (Charles River, Saint-Germain-sur-l'Arbresle, France) were housed at the University of Bordeaux animal facility A2, according to the rules and regulations of the Institutional Animal Care and Use Committee (agreement number B33-063-916). Pancreatic cell lines origins have been described earlier (10). Genomic KRAS PCR amplification and sequencing were carried out to control the identity of the cells (11). Their homozygous or heterozygous status WT or mutant towards KRAS mutation was confirmed on a regular basis on sampled genomic DNA by droplet digital PCR (ddPCR ${ }^{\text {TM }}$ KRAS G12/G13 Screening Kit, Bio-Rad, Marnes-la-Coquette, France). Human primary fibroblasts were obtained from mammoplasties after the patients had given written consent. Cell culture 
was carried out as already described (9). Resveratrol (R), and capsaicin (C) were from Sigma Aldrich. Highly concentrated solutions ( $200 \mathrm{mM}$ each) were prepared in ethanol.

\subsection{In vitro cell survival assay and cell irradiation}

Cells were plated at $7 \times 10^{3}$ cells per well in 96-well plates. The day after, resveratrol and capsaicin alone or in combinations at their IC50, IC50/2 or IC50/4 were applied in the morning. Plates were irradiated with 2-8 Gy delivered by a linear accelerator (Elekta ${ }^{\mathrm{TM}}$ ) using 6MV photons. Cells were kept in culture for an additional $72 \mathrm{~h}$ before cell viability was measured as already described (9). Each point was done at least in quadruplet. Each experiment was repeated at least twice.

\subsection{Protein extraction and western-blotting}

Protein extracts from tumors of xenografted Capan-2 cells and western blotting were performed as already described (10). The following primary antibodies were used: anti-Phospho-ATM, anti-Phospho$\gamma$ H2A.X, anti-Phospho-p53(ser15), anti-Phospho-Chk1, anti-phospho-Chk2, anti-Bax, anti-Bcl2 (Cell Signaling).

\subsection{ROS detection after irradiation}

For ROS detection, cells were seeded in 12 -well plate $\left(2 \times 10^{5}\right.$ cells per well), $24 \mathrm{~h}$ before the experiment. Cells were washed in PBS and incubated for 30 minutes at $37^{\circ} \mathrm{C}$ with Dichloro-dihydro-fluorescein diacetate (DCFH-DA) (DCFDA Cellular ROS Detection Assay, Abcam, 113851), then treated with single BFCs or combinations at their IC50 and/or irradiated with a 6Gy dose. Percentages of fluorescent cells were examined by flow cytometry (BD FACSCanto II Flow Cytometer \#338962).

\subsection{Xenografts of the Capan-2 cells}

Mice were injected subcutaneously with $8 \times 10^{5}$ cells in $100 \mu$ serum-free medium in the right and the left flanks. When the tumors reached about $100 \mathrm{~mm}^{3}$, mice were given BFCs or vehicle by gavage, 3 
times a week. Resveratrol dose was $50 \mathrm{mg} / \mathrm{kg}$ and capsaicin dose was $5 \mathrm{mg} / \mathrm{kg}$. Control mice were administrated with ethanol ( $2 \% \mathrm{v} / \mathrm{v}$ in PBS). The same day mice were fed the BFCs, they were irradiated with 2Gy per fraction delivered using $6 \mathrm{MV}$ photons on a linear accelerator (Elekta ${ }^{\mathrm{TM}}$ ). Treatment tolerance was evaluated with the Lloyds' distress animal scoring system(12). Resected tumors were weighed between 19 and 21 days after starting the treatments.

\subsection{Histology and morphometric analysis}

Tumors were collected, fixed in $10 \%$ normal buffered formalin, and sectioned ( $4 \mu \mathrm{m}$ sections) after paraffin embedding. Slides (10 slides per group) were stained with trichrome masson stain to highlight fibrosis (appearing green). on. Pictures were taken at X100 magnification, and percentages of fibers were evaluated by 2 distinct operators. Pictures were taken under an inverted Nikon Microscope (Eclipse Ti Nikon, Champigny sur Marne, France). They were treated the NIS-Elements Nikon software, connected with a video camera. Tumor necrosis and atypical nuclei were determined by a trained pathologist of Bordeaux University Hospital (GB) (10 slides per group). Cellularity was quantified on fluorescence images by counting the number of nuclei (stained with DAPI) per field ( $n=1566, n=1853, n=1577$, for CT, RT and RT+BFCs groups, respectively). KI67 stain was performed by immunohistochemistry with antiKIG7 primary antibody (Leica) and anti-mouse secondary antibody (Dako). Number of KI67-positive nuclei over total nuclei was counted on pictures (total cell number $n=1746, n=877, n=1385$, for $C T$, RT and RT+BFCs groups, respectively).

\subsection{Statistical analysis}

Statistical tests were performed using the Graph-Pad Prism software (6.04, GraphPad). In vitro results are expressed as mean $\pm S D$, analyzed by unpaired, bilateral Student's $t$ tests. Results in vivo are expressed as mean \pm SEM, analyzed by 2-tailed Mann-Whitney test or unpaired t test with Welch's correction (fibrosis and necrosis). 


\section{RESULTS}

\subsection{Combination of BFCs radiosensitizes radiosensitive pancreatic tumor cells in vitro}

To assess tumor cell line sensitivity to X-rays irradiation, the Capan-2 cells were irradiated with increasing doses of X-rays and cell survival was determined 48,72 or $96 \mathrm{~h}$ after irradiation. The maximal doseresponse cytotoxicity was obtained $72 \mathrm{~h}$ after irradiation (Figure 1A). Next, we found that Capan-2 and BxPC3- cells were sensitive to irradiation with lethal dose 50 (DL50) around 4Gy and 6Gy respectively. By contrast, PANC-1 and Mia PaCa-2 cells were radioresistant (Figure 1B).

To determine whether irradiation and combination BFCs (resveratrol and capsaicin) could have additive effects, we determined the best combination concentration allowing further measurable radiotoxicity. We chose to work on one radiotherapy-sensitive cell line to (Capan-2) and one resistant cell line (PANC1). BFC combination cytotoxicity using resveratrol and capsaicin inhibitory cytotoxic dose 50 (IC50) was too strong to see any additive effect of irradiation. We tested combination treatment at IC50/2 and IC50/4 and found that the best option was to treat the cells with BFCs at half of their IC50, leading to about $50 \%$ cell cytotoxicity ( $p<0.001$ as compared to control condition). Next, we treated the cells with BFCs (IC50/2), or BFCs alone (IC50) and radiotherapy (RT, 6Gy). For Capan-2 cells, we found that each BFC used alone could significantly sensitize cells to RT (Figure 2, $p<0.001$ when comparing RT alone to RT $+\mathrm{R}$ or $\mathrm{C}$ ). Combination treatment, even at IC50/2 had a significant stronger effect $(\mathrm{p}<0.001$ when comparing BFCs and BFCs+RT). For the PANC-1 cell line, combination of BFCs without RT was toxic, however no radiosensitization was evidenced.

Thus, RT cytotoxic effect was increased by combination of BFCs for radiosensitive cells but not for radioresistant cells in vitro.

3.2. Combination of BFCs increases radio-induced reactive oxygen species (ROS) production in vitro 
We previously demonstrated that combination treatment increased ROS production in Capan-2 cells (9). As X-Rays irradiation induces the production of ROS, we hypothesized that combination treatment of BFCs and RT could exacerbate ROS production. We treated cells in vitro with BFCs and RT and ROS induction was quantified as percentage of fluorescent cells (Figure 3). As expected, RT induced significant increase of ROS producing cells ( $p<0.01$, as compared to control condition) and BFCs further enhanced this effect ( $p<0.01$ as compared to RT alone). Importantly the same observations were made in two different pancreatic cell lines (Capan-2 and BXPC-3). Mean fluorescence intensities were significantly increased after RT treatment, in the presence of BFCs, for Capan-2 cells (not shown).Thus, BFCs increased ROS production resulting from irradiation.

\subsection{Combination treatment of RT and BFCs was efficient to inhibit tumor progression in vivo}

We have already shown that the $R+C$ combination diminished tumor progression when used alone (9). To test whether the combined toxic activity of BFCs and RT was efficient in growing tumors, mice bearing subcutaneous pancreatic tumors from Capan-2 xenografts were treated with RT or RT + BFCs. Tumor masses were significantly lower for RT and RT+BFCs groups compared to control group $(p=0.006$ and $p=0.001$, respectively, Figure $4 A$ ) and tended to be smaller in the $R T+B F C$ s group compared to RT group. We found that tumor progression was significantly slower in the RT+BFCs group as compared to the control group $(p<0.001)$, and to a lesser extent in the RT group. Interestingly, RT+BFCs effect was significantly higher than RT alone, leading to stagnation of tumor growth (Figure 4A), and a tumor volume difference of $40 \%(p<0.05)$.

To further explore treatment impact on tumor progression, we analyzed histologic sections and performed morphometric analyses. Tumors treated by RT or RT+BFCs displayed increased fibrosis, significantly different from control tumors, suggesting that RT induced desmoplastic reaction (Figure 5A a, e, i and d, h, l). Treated tumors also underwent significant enhanced necrotic reaction as compared to control group, suggesting that treatment was efficient (Figure 5B). However, addition of BFCs did not 
modulate changes seen after RT treatment. Moreover, RT-treated group displayed more numerous atypical nuclei (66\% of 12 slides) than RT+BFC or control groups ( $40 \%$ of 10 and 8 slides, respectively). Furthermore, cellularity diminished, especially in the RT+BFCs group, with nuclei displaying condensed chromatin as observed in apoptotic cells. KI67 staining, revealing cycling cells, showed that CT and RT+BFCs tumor groups had similar proportion of cells active in the cell cycle as compared to tumors treated with RT only showing a significant decreased in KI67-positive nuclei (Figure 5A c, g and k).

Taken together these results showed a modest effect of BFC addition in RT treatment, leading to keep cells cycling and potentially inducing chromatin condensation.

\subsection{BFCs modified molecular tumor response to RT treatment}

X-Ray radiations induce diverse lesions in cells, involving radiolysis-derived ROS including DNA damages. To understand molecular responses to RT of treated tumors, we performed western blotting of proteins extracts from CAPAN-2 xenografted tumors, focusing on proteins involved in double stranded break repair pathway. One of the earliest events recruits ataxia telangiectasia mutated (ATM) to the DNA break, which phosphorylates histone $\gamma \mathrm{H} 2 \mathrm{~A}$.X at serine 139 (Figure 6A) (13). Control tumors displayed low levels of phospho-H2A.X, whereas RT treated tumors showed a massive increase as compared to control group (about 40 -fold, $p<0.01$, Figure $6 B$ ). Surprisingly, addition of BFCs with RT strongly inhibited $\gamma \mathrm{H} 2 \mathrm{~A} . \mathrm{X}$ phosphorylation $(p<0.05)$, keeping its level similar as that observed for control tumors. To investigate this unexpected result, we looked at ATM autophosphorylation (Ser1981), which is necessary for ATM kinase activity. BFC treatment diminished ATM phosphorylation as compared to RT alone (Figure 6C, $p<0.05)$. Next, we looked at downstream effectors of the double-stranded break DNA repair pathway. Both checkpoint kinases (Chk), Chk1 and Chk2 were activated by phosphorylation after RT treatment, with or without BFCs, similarly (Figure 6D). Phosphorylation of p53 (Ser15) was significantly increased in RT treated tumors with BFCs (Figure 6E). Ratios of BAX/BCL2 proteins increased significantly only in RT+BFC tumors ( $p<0.05$, Figure $6 F)$. 
Cell growth stagnation and cell death can occur by senescence and necroptosis induction, respectively. We evaluated senescence after combination treatment ( $\mathrm{RT}+\mathrm{BFC})$ in vitro, by acidic beta-galactosidase stain and found very few beta-galactosidase-positive cells in all tested conditions (data not shown). Western blotting of proteins involved in necroptosis cascade such as phospho-RIP3 and phospho-MLKL found no differences between groups (not shown).

Thus, these results suggest that BFC addition to RT opposes RT-induced cell cycle arrest and precipitates cells in apoptotic death.

\section{DISCUSSION}

Although bioactive food components have caught attention for chemosensitization, few studies have focused on radiosensitization. As for chemotherapy, tumor cells respond with diverse amplitudes to radiotherapy, as exemplified in our study. We found the pancreatic tumor cell lines BxPC-3 and Capan-2 sensitive, while Mia PaCa-2 and PANC-1 cells were resistant. When considering their genotypes (14), PANC-1 and Mia PaCa-2 are both mutated on KRAS, TP53 and CDKN2A/p16, while wild type (WT) for SMAD4. BxPC-3 and Capan-2 are both WT for $p 16$ and have different mutational status for the 3 other main mutated genes in PDAC. CDKN2A/p16 is a cyclin-dependent kinase inhibitor. Its presence might render tumor cells more vulnerable to ionizing radiations as previously reported (15), especially because CDKN2A/p16 proficient tumor cells may still be able to reactivate $\mathrm{pRB}$ and its antiproliferative properties (16). Alternatively, PANC-1 and Mia PaCa-2 both retains valid SMAD4 gene, but this may not be involved in RT resistance since SMAD4 re-expression in Pancreatic tumor cells does not seem to reactivate SMAD4 inhibitory pathways, especially when cells have evolved through epithelial to mesenchymal transition (EMT) (18). We and others have previously shown that PANC-1 cells have undergone EMT, and they also carry more cancer stem cell (CSC) like cells, which could confer acute resistance to radiotherapy (17).

Several BFCs radiosensitize tumor cells such as the polyphenols curcumin (18) and quercetin (19) or the sulfamide sulforaphane (20), for example. Considering our previous work on pancreatic tumor cell chemosensitization by combination of capsaicin and resveratrol (9), we tested the same approach with radiotherapy. We found that ionizing radiations in vitro cytotoxicity was amplified by BFC addition, at two different doses (IC50 or IC50/2 for resveratrol or capsaicin) for the Capan-2 cell line. By contrast, PANC-1 cells, although sensitive to BFC treatment were resistant to radiotherapy. BFCs did not sensitize them to RT. This suggests that radioresistance relies on specific capacity of PANC-1 towards 
radiotherapy. Others have reported failure in radiosensitizing the PANC-1 cell line (21). This resistance may depend on the expression level of the apoptosis inhibitor survivin $(21,22)$. Alternatively, as already mentioned above, PANC-1 cells may carry intrinsic radioresistance linked to their CSC-like phenotype, that could not be counteracted by BFC addition.

By contrast to PANC-1 response, Capan-2 cells were more sensitive to radiotherapy in the presence of BFCs. Increased cell toxicity was observed when cells were irradiated and treated with a single BFC at their specific IC50 and maintained when resveratrol and capsaicin were used together, even at half their IC50. Several reports showed that resveratrol as well as capsaicin carry tumor cell toxicity by increasing ROS levels (23-25). When starting this study, we predicted that ROS production by different pathways and by converging actions of resveratrol and capsaicin and radiotherapy could increase the amplitude of ROS-induced DNA damages. We showed in vitro that ionizing radiations induced ROS producing cells with two different cell lines. The percentages of ROS producing cells was further increased in the presence of BFCs. This is in agreement with published work showing a ROS-dependent radiosensitization of colon cancer cells by resveratrol (26).

We performed in vivo evaluation of the combination treatment RT+BFCs in a subcutaneous xenograft model, with BFC administration by gavage and a full body irradiation program. Tumor volume analysis confirmed significant radiosensitization by BFCs. We observed a trend of lower mean masses that could reach significance if the experiments were continued over a longer period. However, total body irradiation resulted in aplasia, which prevented further irradiations. Alternatively, similar tumor masses could still carry difference only visible at the microscopic level. To address this question, we analyzed sections of tumors within all the groups. We found that fibrotic reaction and necrosis were similarly increased in RT-treated groups, with or with BFCs, underlying efficiency of the treatments. This suggests that RT might be the main effector of tumor toxicity with a minimal modulation by BFCs. Moreover, we found a modest diminution of cellularity in the RT+BFCs treated group that could account for the observed loss of mass as compared to control.

As already reported by us and others, resveratrol or capsaicin treatment, as well as both combined induced high levels of apoptosis, involving several intracellular factors leading to unbalanced pro/antiBCL2 members $(9,27,28)$. We found the same impact in the present study in tumor treated with RT+BFCs, further confirmed with decreased cellularity and chromatin compaction visualized on histological section. Interestingly, RT alone did not change significantly BAX/BCL2 ratio as compared to control tumors. In fact, RT treatment alone led to significant increase in BCL2 levels (Figure 5, $p<0.01$ ), 
and a trend to diminish BAX levels. Irradiated Mia PaCa-2 cells also changed their BAX to BCL2 balance in the same way (20), the control of BCL2 family member levels being known as a mechanism of RT resistance (29). Our result suggests that addition of BFCs to irradiation counteracts this resistance mechanism.

The gene suppressor p53 is at the crossroad between apoptosis induction and cell cycle arrest for DNA repair (Figure 5A). We found an increase in phospho-stabilization of p53 on ser15 (and also ser6 and ser9, not shown) in RT+BFC group. Checkpoint Kinases 1 and 2 observed activation probably resulted in p53 phosphorylation. When p53 is stabilized by phosphorylation, it first stops the cell cycle by CKI production. Interestingly, the proliferation marker KI67 was turned down in RT only treated tumors and was maintained as in controls in the presence of BFCs. To explain this combination of results we can hypothesize that RT induces cell cycle arrest, which might be counteracted by BFCs, precipitating tumor cells in unrepaired mitosis, and exacerbating intrinsic apoptosis. This effect was previously described for resveratrol treatment (30). Moreover, capsaicin induced apoptosis in irradiated prostate cancer cells by opposing NFkB survival signal, independently of cell cycle arrest (31).

Next, we searched for BFC impact on RT response in earlier stages of DNA damage response. Importantly, we found profound differences between control and treated groups, and more surprisingly between RT alone and RT+BFCs groups. As the first hypothesis was that increased ROS might induce enhanced DSBs, we looked at $\gamma \mathrm{H} 2$.AX phosphorylation, which is one of the first steps of DSB DNA repair response (13), witnessing its beginning. Strikingly, and unexpectedly, $\gamma \mathrm{H} 2 . \mathrm{AX}$ phosphorylation displayed a sharp increase after RT treatment, completely annihilated in the presence of BFCs. We found that this was probably due to inhibition of ATM activation, since ATM phosphorylation was no more detectable in the presence of BFCs. Resveratrol alone enhances IR-induced DNA-damage response signals, probably by ROS-related DSBs, leading to ATM activation and radiosensitization of prostate cancer cells (30) or in chronic treatment. Moreover, resveratrol alone can directly activate ATM (32). In the same way, capsaicin is known to induce ROS production and thereby DNA damages (25), but no direct action has been described on ATM activation. Interestingly, combination of resveratrol and capsaicin leads to strong ATM inhibition, as shown in the present study. This is in agreement with the inhibitory action of resveratrol on DNA repair expression members, including ATM in MCF7 cells by downregulating their expression (33). Resveratrol also exerted global homologous repair and non-end joining DNA repair inhibition in an ATM-p53-dependent pathway (34). In the same way, the other polyphenol curcumin inhibited ATM in irradiated tumor kidney cells (35). Importantly, ATM-deficient pancreatic tumor cells 
were more susceptible to ionizing radiation than ATM-proficient cells (36). All these data concur with the present results. So, in light of our data and published results, we hypothesize that, in one hand, additive ROS production by RT and BFCs induce ROS-related DSBs. Normal RT-related ATM-dependent DNA repair pathway is inhibited by BFCs, including DNA lesions recognition by ATM and cell cycle arrest. In the other hand, p53-related apoptosis induction is maintained and majored by cell cycle maintenance (Figure 7).

Despite ATM inhibition, we found increased phosphorylation of the downstream checkpoint kinases $\mathrm{CHK} 1$ and $\mathrm{CHK} 2$. According to what is known in the cascade of events induced by RT, and as summarized in Figure 7, it si possible that ATR (ATM and Rad3-related) and DNA-PKc activities are maintained in the presence of BFCs and trigger p53-dependant apoptotic response. This is further supported by the fact that ATM is the major actor of $\gamma \mathrm{H} 2$.AX phosphorylation, ATR and DNA-PKcs carrying only limited activity (13).These points need further validation.

In conclusion, future studies are needed to optimize RT + BFCs combined effects as compared to RT used alone, by increasing the frequency of BFC treatments, or majoring their bio-availability with more systemic administration. Another option currently under investigation in our lab is to perform focal irradiation delivering high doses to improve RT-related toxicity. Nonetheless, we are describing interesting mechanistic aspects of resveratrol and capsaicin combined action on DNA repair pathway.

\section{ACKNOWLEDGEMENTS}

The authors would like to thank Katia Obama and Isabelle Lamy for administrative assistance and fund management, and the staff of University of Bordeaux animal facility, in particular Julien Izotte for technical assistance.

\section{FUNDING SOURCES}

This work was supported by grants from the Ligue régionale contre le cancer (Comité Dordogne), France.

\section{REFERENCES}

1. Cowppli-Bony A, Uhry Z, Remontet L, Voirin N, Guizard A-V, Trétarre B, et al. Survival of solid cancer patients in France, 1989-2013: a population-based study. Eur J Cancer Prev. nov 2017;26(6):461-8. 
2. Rahib L, Smith BD, Aizenberg R, Rosenzweig AB, Fleshman JM, Matrisian LM. Projecting Cancer Incidence and Deaths to 2030: The Unexpected Burden of Thyroid, Liver, and Pancreas Cancers in the United States. Cancer Res. 1 juin 2014;74(11):2913-21.

3. Kim BM, Hong Y, Lee S, Liu P, Lim JH, Lee YH, et al. Therapeutic Implications for Overcoming Radiation Resistance in Cancer Therapy. Int J Mol Sci. 2014;16(11):26880-913.

4. Waddell N, Pajic M, Patch A-M, Chang DK, Kassahn KS, Bailey $P$, et al. Whole genomes redefine the mutational landscape of pancreatic cancer. Nature. 2015;518(7540):495-501.

5. Seshacharyulu P, Baine MJ, Souchek JJ, Menning M, Kaur S, Yan Y, et al. Biological determinants of radioresistance and their remediation in pancreatic cancer. Biochim Biophys Acta BBA - Rev Cancer. août 2017;1868(1):69-92.

6. Zhu C-P, Shi J, Chen Y-X, Xie W-F, Lin Y. Gemcitabine in the chemoradiotherapy for locally advanced pancreatic cancer: A meta-analysis. Radiother Oncol. mai 2011;99(2):108-13.

7. Chand S, O'Hayer K, Blanco FF, Winter JM, Brody JR. The Landscape of Pancreatic Cancer Therapeutic Resistance Mechanisms. Int J Biol Sci. 2016;12(3):273-82.

8. Boreddy SR, Srivastava SK. Pancreatic cancer chemoprevention by phytochemicals. Cancer Lett. 2013;334(1):86-94.

9. Vendrely V, Peuchant E, Buscail E, Moranvillier I, Rousseau B, Bedel A, et al. Resveratrol and capsaicin used together as food complements reduce tumor growth and rescue full efficiency of low dose gemcitabine in a pancreatic cancer model. Cancer Lett [Internet]. [cité 13 janv 2017]; Disponible sur: http://www.sciencedirect.com/science/article/pii/S0304383517300174

10. Lafitte M, Moranvillier I, Garcia S, Peuchant E, lovanna J, Rousseau B, et al. FGFR3 has tumor suppressor properties in cells with epithelial phenotype. Mol Cancer. 2013;12(1):1.

11. Wang Z, Desmoulin S, Banerjee S, Kong D, Li Y, Deraniyagala RL, et al. A comprehensive in vitro characterization of pancreatic ductal carcinoma cell line biological behavior and its correlation with the structural and genetic profile. Mol Anal Inhib Eff N-Acetyl--Cysteine Prolif Invasiveness Pancreat Cancer Cells. 2004;445(3):236-47.

12. Lloyd MH, Wolfensohn SE. Practical use of distress scoring systems in the application of humane endpoints. Hum Endpoints Anim Exp ... [Internet]. 1999; Disponible sur: http://lal.org.uk/uploads/editor/HEP_Lloyd.pdf

13. Burma S, Chen BP, Murphy M, Kurimasa A, Chen DJ. ATM Phosphorylates Histone H2AX in Response to DNA Double-strand Breaks. J Biol Chem. 9 nov 2001;276(45):42462-7.

14. Deer EL, González-Hernández J, Coursen JD, Shea JE, Ngatia J, Scaife CL, et al. Phenotype and Genotype of Pancreatic Cancer Cell Lines: Pancreas. mai 2010;39(4):425-35. 
15. Agarwal $P$, Lutful Kabir FM, Delnnocentes $P$, Curtis R. Tumor Suppressor Gene $p 16 /$ INK4A/CDKN2A and Its Role in Cell Cycle Exit, Differentiation, and Determination of Cell Fate. In: Cheng Y, éditeur. Tumor Suppressor Genes [Internet]. InTech; 2012 [cité 4 sept 2018]. Disponible sur: http://www.intechopen.com/books/tumor-suppressor-genes/tumor-suppressor-gene-p16-ink4acdkn2a-and-its-role-in-cell-cycle-exit-differentiation-and-determina

16. Knudsen ES, Wang JYJ. Targeting the RB-pathway in Cancer Therapy. Clin Cancer Res. 15 févr 2010;16(4):1094-9.

17. Wang L, Li P, Hu W, Xia Y, Hu C, Liu L, et al. CD44+CD24+ subset of PANC-1 cells exhibits radiation resistance via decreased levels of reactive oxygen species. Oncol Lett. août 2017;14(2):1341-6.

18. Sebastià N, Montoro A, Hervás D, Pantelias G, Hatzi VI, Soriano JM, et al. Curcumin and transresveratrol exert cell cycle-dependent radioprotective or radiosensitizing effects as elucidated by the PCC and G2-assay. Mutat Res. 2014;766-767:49-55.

19. Lagerweij T, Hiddingh L, Biesmans D, Crommentuijn MHW, Cloos J, Li X-N, et al. A chemical screen for medulloblastoma identifies quercetin as a putative radiosensitizer. Oncotarget [Internet]. 14 juin 2016 [cité 13 sept 2018];7(24). Disponible sur: http://www.oncotarget.com/fulltext/7980

20. Kubota N. Benzyl isothiocyanate sensitizes human pancreatic cancer cells to radiation by inducing apoptosis. Int J Mol Med [Internet]. 10 août 2011 [cité 3 sept 2018]; Disponible sur: http://www.spandidos-publications.com/10.3892/ijmm.2011.770

21. Galloway NR, Aspe JR, Sellers C, Wall NR. Enhanced Antitumor Effect of Combined Gemcitabine and Proton Radiation in the Treatment of Pancreatic Cancer: Pancreas. oct 2009;38(7):782-90.

22. Asanuma K, Kobayashi D, Furuya D, Tsuji N, Yagihashi A, Watanabe N. A Role for Survivin in Radioresistance of Pancreatic Cancer Cells. Jpn J Cancer Res. sept 2002;93(9):1057-62.

23. Heiss EH, Schilder YDC, Dirsch VM. Chronic Treatment with Resveratrol Induces Redox Stress- and Ataxia Telangiectasia-mutated (ATM)-dependent Senescence in p53-positive Cancer Cells. J Biol Chem. 14 sept 2007;282(37):26759-66.

24. Xie L, Xiang G-H, Tang T, Tang $Y$, Zhao L-Y, Liu D, et al. Capsaicin and dihydrocapsaicin induce apoptosis in human glioma cells via ROS and Ca2+-mediated mitochondrial pathway. Mol Med Rep. nov 2016;14(5):4198-208.

25. Yang K, Pyo J, Kim G-Y, Yu R, Han I, Ju S, et al. Capsaicin induces apoptosis by generating reactive oxygen species and disrupting mitochondrial transmembrane potential in human colon cancer cell lines. Cell Mol Biol Lett [Internet]. 1 janv 2009 [cité 4 sept 2018];14(3). Disponible sur: http://www.degruyter.com/view/j/cmble.2009.14.issue-3/s11658-009-0016-2/s11658-009-00162.xml

26. Tak J-K, Lee J-H, Park J-W. Resveratrol and piperine enhance radiosensitivity of tumor cells. BMB Rep. 30 avr 2012;45(4):242-6.

27. Kim MY, Trudel LJ, Wogan GN. Apoptosis induced by capsaicin and resveratrol in colon carcinoma cells requires nitric oxide production and caspase activation. 2009;29(10):3733-40. 
28. Liu T, Wang G, Tao H, Yang Z, Wang Y, Meng Z, et al. Capsaicin mediates caspases activation and induces apoptosis through P38 and JNK MAPK pathways in human renal carcinoma. BMC Cancer. 2016;16(1):790.

29. Thomas S, Quinn BA, Das SK, Dash R, Emdad L, Dasgupta S, et al. Targeting the Bcl-2 family for cancer therapy. Expert Opin Ther Targets. janv 2013;17(1):61-75.

30. Rashid A, Liu C, Sanli T, Tsiani E, Singh G, Bristow RG, et al. Resveratrol enhances prostate cancer cell response to ionizing radiation. Modulation of the AMPK, Akt and mTOR pathways. Radiat Oncol. 2011;6(1):144.

31. Venier NA, Colquhoun AJ, Sasaki H, Kiss A, Sugar L, Adomat H, et al. Capsaicin: A novel radiosensitizing agent for prostate cancer: Capsaicin as a Radio-Sensitizer. The Prostate. févr 2015;75(2):113-25.

32. Lee J-H, Guo Z, Myler LR, Zheng S, Paull TT. Direct Activation of ATM by Resveratrol under Oxidizing Conditions. Hammond E, éditeur. PLoS ONE. 16 juin 2014;9(6):e97969.

33. Leon-Galicia I, Diaz-Chavez J, Garcia-Villa E, Uribe-Figueroa L, Hidalgo-Miranda A, Herrera LA, et al. Resveratrol induces downregulation of DNA repair genes in MCF-7 human breast cancer cells: Eur J Cancer Prev. janv 2013;22(1):11-20.

34. Gatz SA, Keimling M, Baumann C, Dörk T, Debatin K-M, Fulda S, et al. Resveratrol modulates DNA double-strand break repair pathways in an ATM/ATR-p53- and -Nbs1-dependent manner. Carcinogenesis. mars 2008;29(3):519-27.

35. Li G, Wang Z, Chong T, Yang J, Li H, Chen H. Curcumin enhances the radiosensitivity of renal cancer cells by suppressing NF-KB signaling pathway. Biomed Pharmacother. oct 2017;94:974-81.

36. Ayars M, Eshleman J, Goggins M. Susceptibility of ATM-deficient pancreatic cancer cells to radiation. Cell Cycle. 19 mai 2017;16(10):991-8.

\section{FIGURE LEGENDS}

Figure 1: Pancreatic cancer cells display different radiosensitivities

Cells were irradiated with increasing doses of X-Rays and cell viability was quantified A) according to different times post irradiation in CAPAN-2 cell line B) according to pancreatic tumor cell lines 72 hours post irradiation. 
Figure 2: Combination of resveratrol and capsaicin radiosensitizes radiosensitive but not radioresistant pancreatic tumor cells

Before combination effect of radiotherapy and BFCs was tested, cell viability was determined in the presence of BFCs at different doses: IC50, IC50/2 and IC50/4, for the radiosensitive Capan-2 cells (A) and for the radioresistant PANC-1 cells (B). Both cell lines were then treated with single BFCs (at IC50) or with combination BFCs at IC50/2 each, and a radiation dose of 6Gy. Cell viability was determined $72 \mathrm{~h}$ after irradiation. ${ }^{* * *}: p<0.001, \mathrm{~ns}$ : not significant when conditions with or without irradiation are compared. R: resveratrol, C: capsaicin, RT: radiotherapy, Con: control treated with vehicle. Regardless of RT or Con conditions, ratio of live cells are lower in BFC conditions than without BFC $(p<0.001)$

\section{Figure 3: BFC addition to radiotherapy enhances oxygen reactive species production}

Cells were incubated for 30 minutes at $37^{\circ} \mathrm{C}$ with DCFH-DA, then treated with radiotherapy (RT), or combination treatment of BFCs (resveratrol and capsaicin) and RT or were treated with vehicle (CON). Percentages of fluorescent cells increase, marking the production of ROS was quantified by flow cytometry. A) Representative dot plots are shown for each condition, with \% of fluorescent cells indicated in the positive window. B) Individual results of percentages of fluorescent cells were plotted and mean \pm SD are shown for each condition, for two different cell lines (Capan-2 and BCPC-3 cells). **: $p<0.01, * * *: p<0.001$

Figure 4: BFC addition to radiotherapy modulates xenografted tumor RT response

Capan-2 cells were injected subcutaneously in nude immune-deficient mice. When tumors reached about $100 \mathrm{~mm} 3$, mice received BFCs in combination by gavage and/or were irradiated (2 Gy whole body treatment) 3 times a week, for 3 weeks. Control group ( $C O N n=12$ individual tumors) received vehicle orally. $\mathrm{RT}$ group received vehicle orally ( $n=23$ individual tumors). BFC $+R T$ group received a combination of resveratrol $50 \mathrm{mg} / \mathrm{kg}(\mathrm{R})$ and capsaicin $5 \mathrm{mg} / \mathrm{kg}$ (C) ( $\mathrm{n}=18$ individual tumors). (A) Tumor masses are 
represented as mean \pm SEM calculated from pooled experiments, as compared to the control group mean masses. (B) Tumor volumes are represented as mean \pm SEM calculated from pooled experiments, as compared to the control group mean volume. . ${ }^{*} p<0.05,{ }^{* *} p<0.01,{ }^{* *} p<0.001$, ns: not significant by a two-tailed Mann Whitney test.

\section{Figure 5: BFC addition to radiotherapy keeps tumor cells cycling}

(A) Tumors were collected, fixed and embedded in paraffin. Sections were stained by Trichrome Masson (a, e, i), original magnification X100. DAPI and KI67 staining are shown for each condition $(b, f, j$ and $c, g$, k respectively) original magnification $\mathrm{X} 200$ and $\mathrm{X} 400$, respectively. Hematoxylin and eosin (H\&E) stain shows tumor structure $(d, h, I)$ original magnification $\mathrm{X} 400$. Fibrosis is visualized by long green fibers in between the epithelial tumor clusters. (B) Mean \pm SEM tumor fibrosis and necrosis quantifications are displayed. Mean \pm SEM cellularity and KI67 positive nuclei are presented. Yellow arrows point atypical nuclei whereas red arrows show typical DNA-containing apoptotic vesicles. Fibrosis was evaluated by 2 distinct operators, as percentages of fibers in microscopic fields (10 slides per group). Tumor necrosis and atypical nuclei were determined by a trained pathologist of Bordeaux University Hospital (GB, 10 slides per group).

Figure 6: BFC addition to radiotherapy results in striking inhibition of DSB repair pathway favoring cell death response in xenografted tumors

(A) Molecular response to radiotherapy: exposure to ionizing radiations induces DNA double-stranded breaks (DSB), leading to DNA damage repair pathways activation with cell cycle arrest and eventually apoptosis induction. Western blotting was performed for at least 3 tumors from xenografted Capan-2 cells of each condition. Densitometry analysis are presented under each blot as a ratio of control condition. Statistical analysis was performed for each quantification: ${ }^{*} p<0.05,{ }^{* *} p<0.01,{ }^{* * *} p<0.001, n s$ : not significant by a two-tailed Mann Whitney test. (B) Immunoblot with anti-phospho-ser139- $\gamma \mathrm{H} 2$.AX. (C) 
Immunoblot with anti-phospho-ser1981-ATM. (D) Immunoblot with anti-phospho-ser345-CHK1 and antiphospho-Thr68-CHK2. (E) Immunoblot with anti-phospho-ser15-p53. (G) Immunoblot with anti-bcl2 and anti-bax. BFC: resveratrol + capsaicin, RT: radiotherapy, Con: control treated with vehicle

Figure 7: Working model of molecular modulation of radiotherapy response by combination treatment of resveratrol and capsaicin

Proposed actions exerted by combination of resveratrol and capsaicin in RT treated xenografted pancreatic tumors according to known DNA damages pathways. Steps modulated by resveratrol and capsaicin together are identified by REV in a green circle and CAP in an orange triangle, respectively. Steps characterized with dashed lines have not been explored in the present study. Cell scheme was downloaded from Servier Medical Art, licensed under a Creative Common Attribution 3.0 Generic License. http://smart.servier.com/. Red arrows delineate induction and red lines with bar end symbolize inhibition.

\section{AUTHOR'S CONTRIBUTIONS}

VV: conception, design, analysis, assembly and interpretation of data, manuscript writing.

SA: collection and assembly of data, interpretation of data.

$\mathrm{CN}$ : collection and assembly of data, interpretation of data.

IM: design, collection and assembly of data.

IL: collection and assembly of data.

BR: design, collection of data.

SC: radiotherapy dose and course validation, collection of data

$A B$ : design, analysis and interpretation of data, final approval of the manuscript.

FMG: design, financial support, final approval of the manuscript. 
EB: financial support, final approval of the manuscript.

GB: analysis of data.

LC: design, interpretation of data and final approval of the manuscript.

CD: design, interpretation of data and final approval of the manuscript.

SD: conception, design, analysis, assembly and interpretation of data, financial support, manuscript writing. 
A

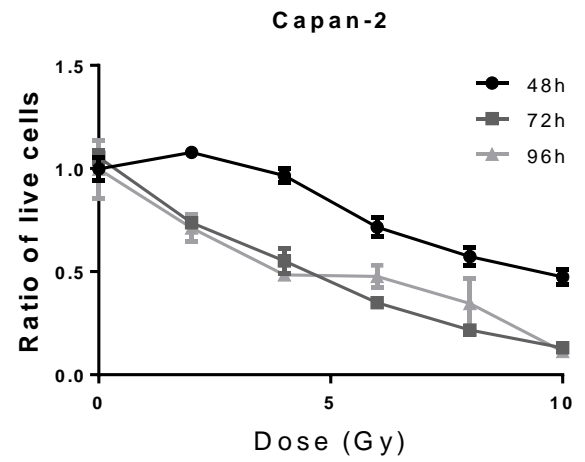

B

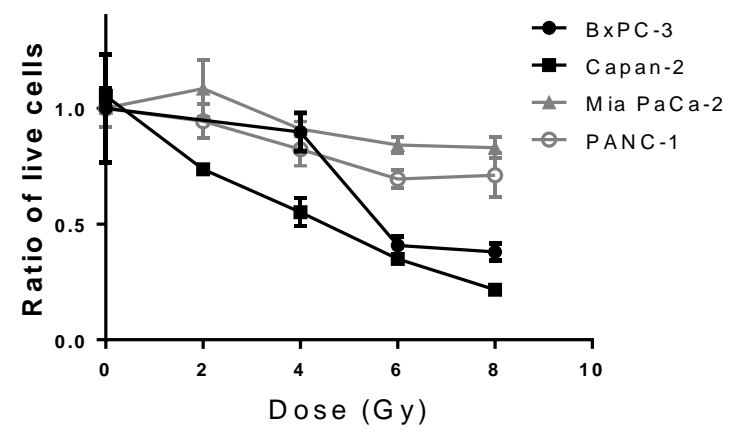

Figure 1 

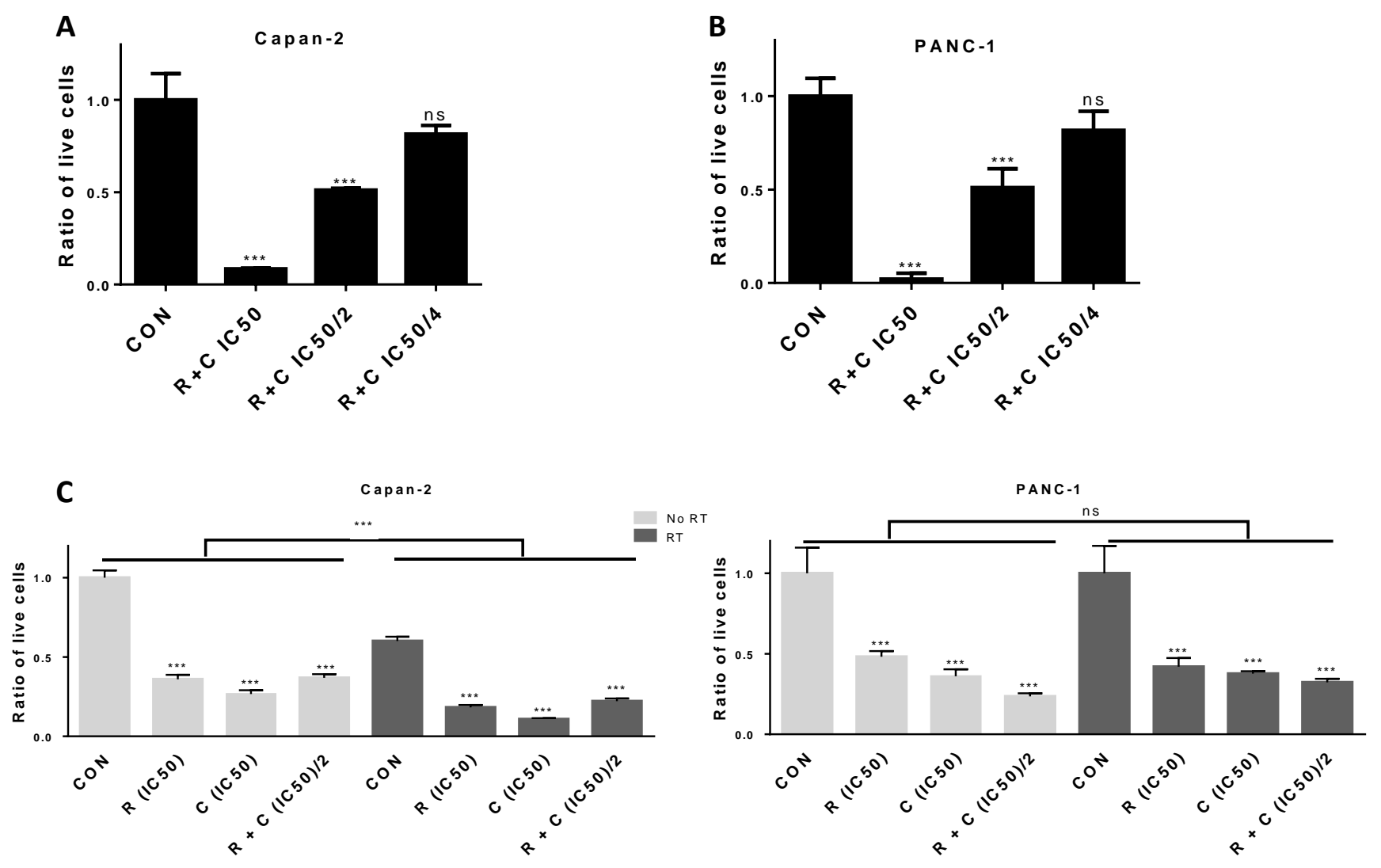

Figure 2 
A
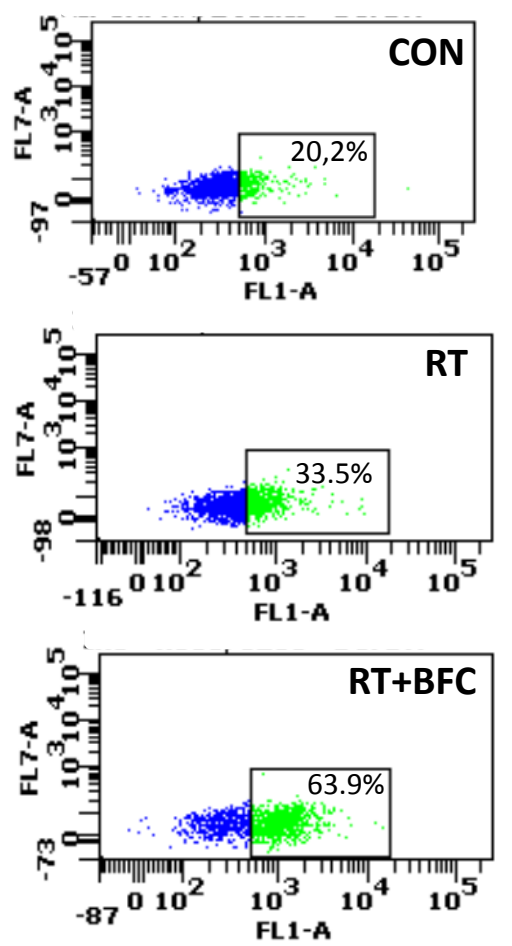

Figure 3
B

C a p a n-2

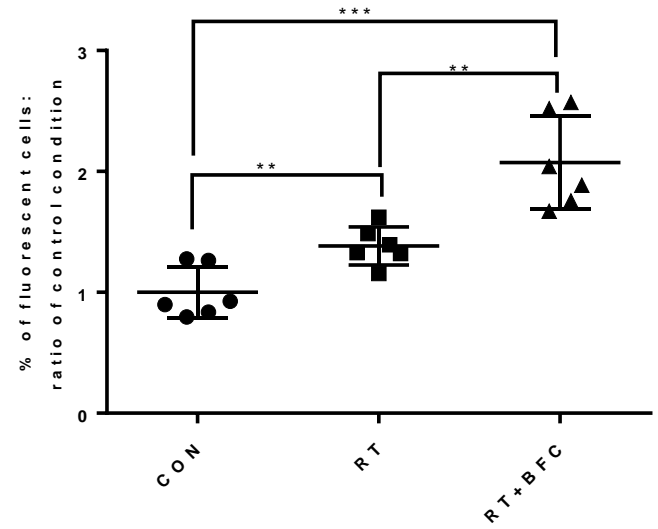

C

B X P C - 3

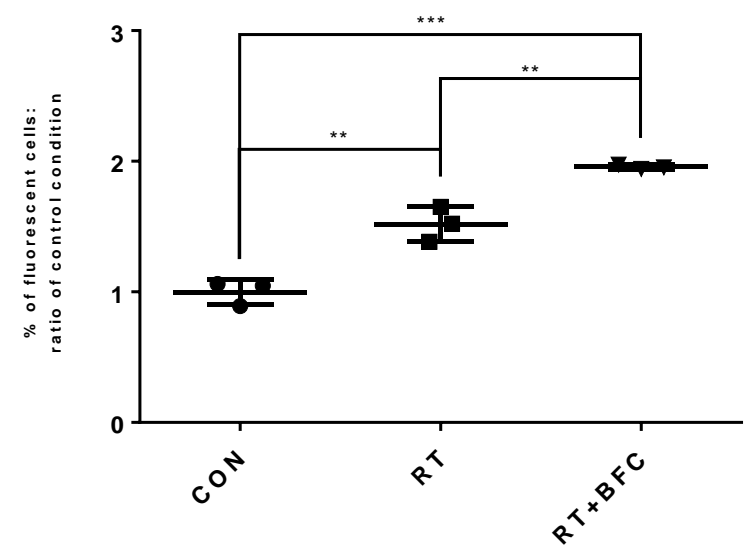


A

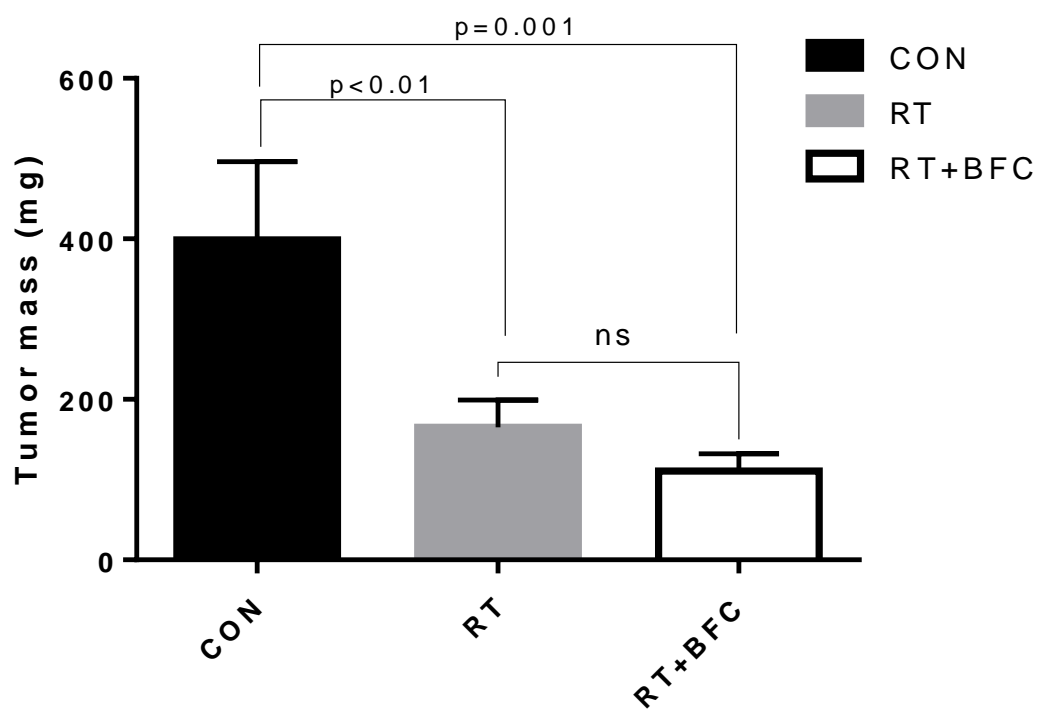

B

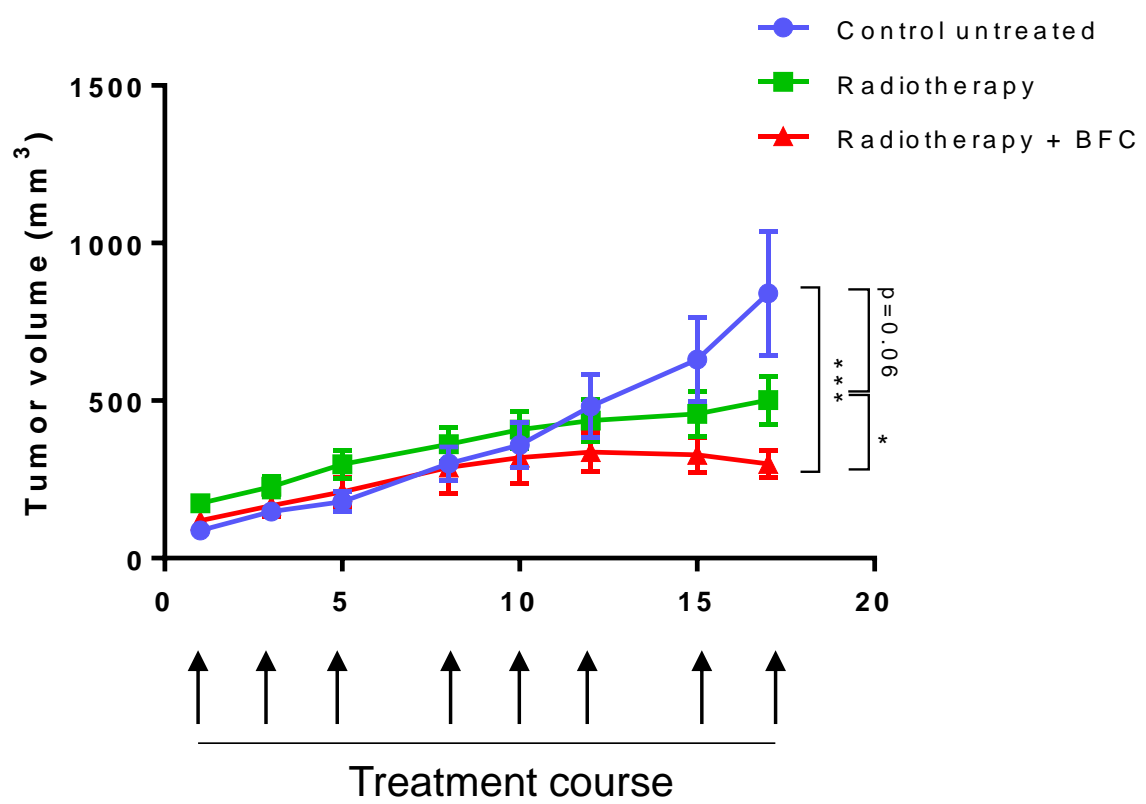

Figure 4 

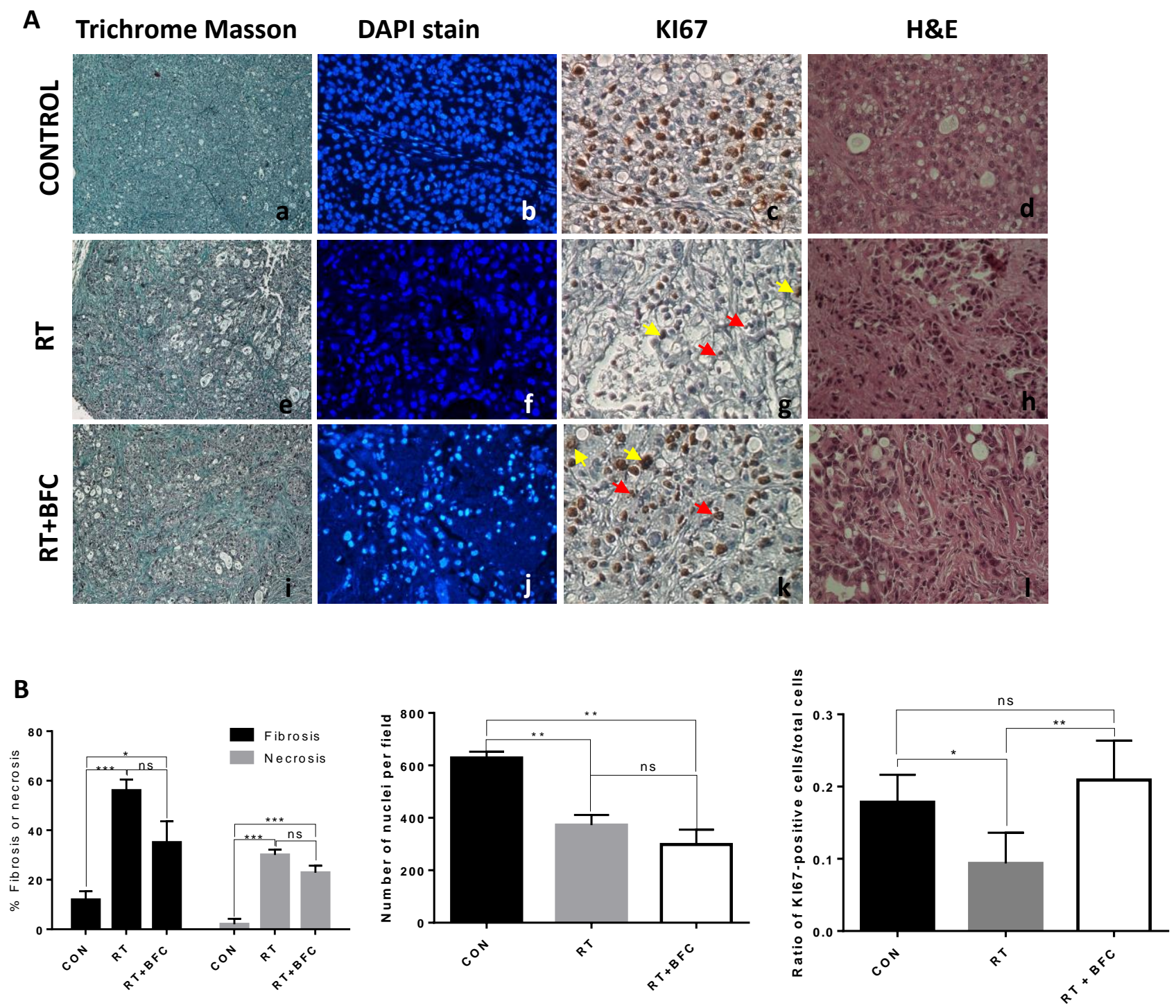

Figure 5 


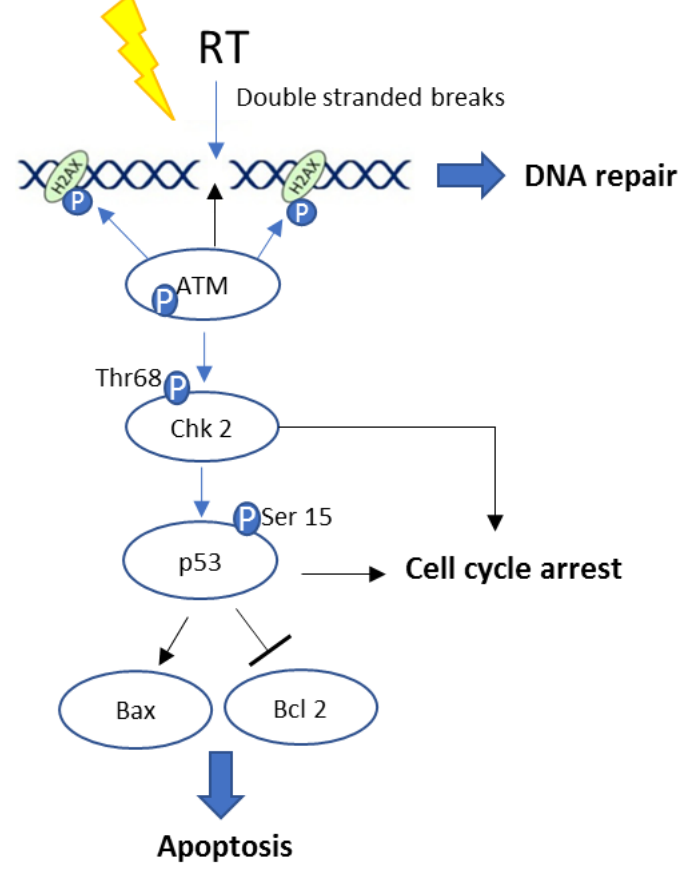

B



$\begin{array}{llll}\text { C } & \mathrm{CON} & \mathrm{RT} & \mathrm{RT}+\mathrm{BFC}\end{array}$
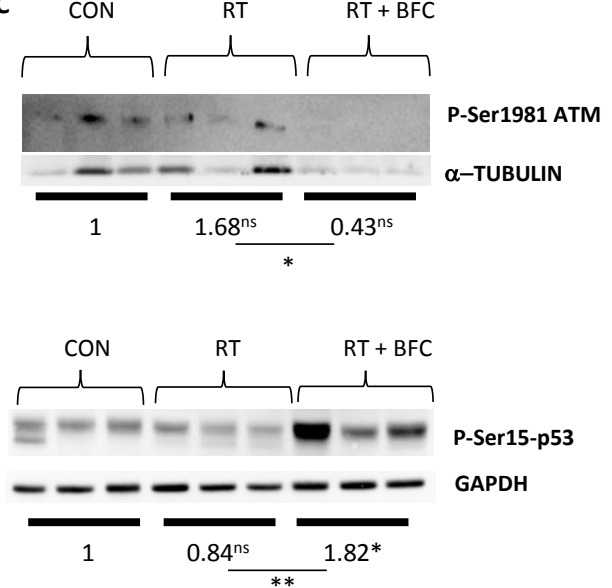

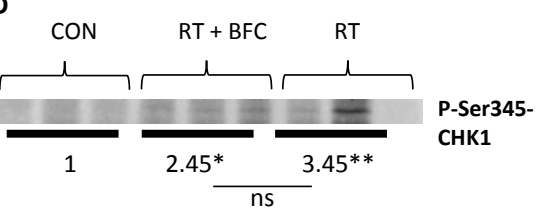
\begin{tabular}{lll}
\hline P-Thr68 & CHK2 \\
\hline
\end{tabular} $------1-$ GAPDH

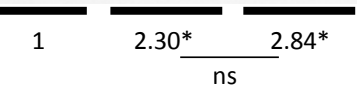

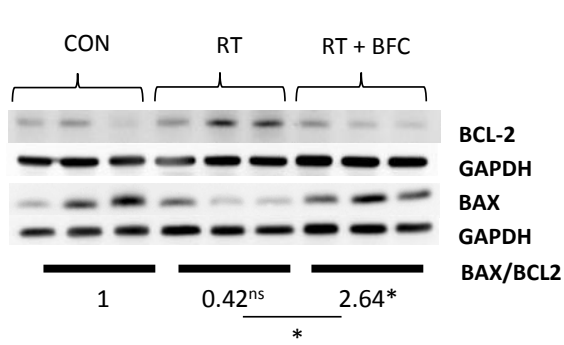

Figure 6 


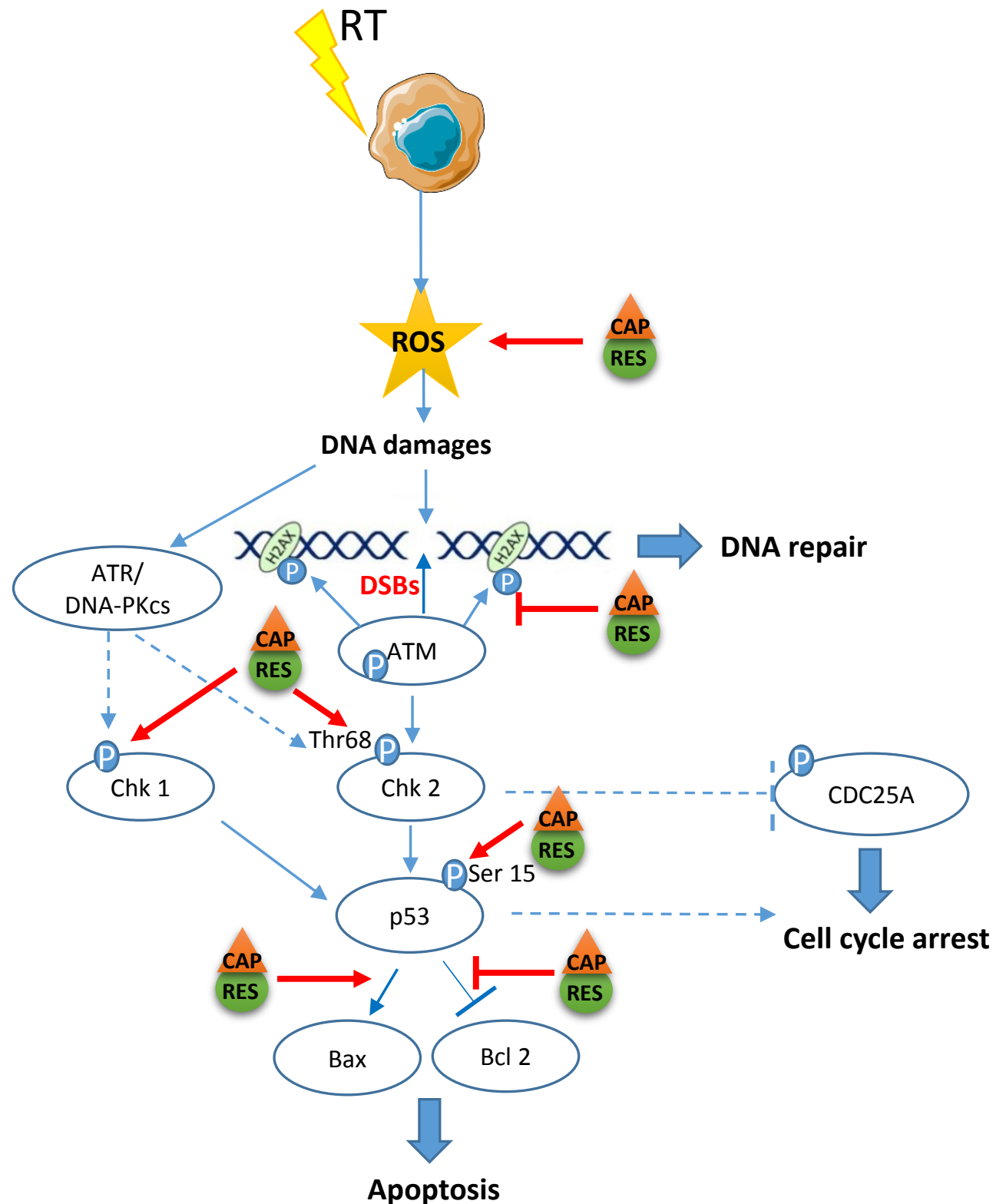

Figure 7 